\title{
Decrease in the Spatial Dispersion at the Termination of Atrial Fibrillation by Intravenous Cibenzoline
}

\author{
Hiroyuki Kakugawa, MD; Akihiko Shimizu, MD*; Toshihiko Yamagata, MD; \\ Masahiro Esato, MD; Takeshi Ueyama, MD; Yasuhiro Yoshiga, MD; \\ Masashi Kanemoto, MD; Masunori Matsuzaki, MD
}

\begin{abstract}
Atrial electrograms were recorded from the high right atrium, coronary sinus, and right lateral wall in 15 patients with induced atrial fibrillation (AF). Intravenous cibenzoline terminated AF in 8 patients (T group), but not in 7 patients (non-T group). The cycle length of the AF (AFCL) obtained by the autocorrelation function was measured every $5 \mathrm{~s}$ during the $30 \mathrm{~s}$ prior to the cibenzoline administration, and just before the termination of $\mathrm{AF}$ or at the end of the cibenzoline infusion in the non-T group. The mean AFCL, and spatial and temporal dispersion of the AFCL were then compared between the 2 groups (dispersion=standard deviation $\times 100 /$ mean AFCL). Cibenzoline significantly increased the mean AFCL and decreased the spatial dispersion in both groups. No significant difference in either the mean AFCL or temporal dispersion before or after cibenzoline was observed between the 2 groups. In addition, no significant difference in the spatial dispersion before the cibenzoline was observed, but the spatial dispersion after the cibenzoline was significantly smaller in the T group than in the non$\mathrm{T}$ group. The mean AFCL, and the spatial and temporal dispersion before the cibenzoline did not predict the termination of AF. The decrease in the spatial dispersion may be the most important mechanism by which intravenous cibenzoline terminates AF. (Circ J 2003; 67: 810-815)
\end{abstract}

Key Words: Atrial fibrillation; Autocorrelation function; Cibenzoline; Spatial dispersion; Temporal dispersion

$\Delta$

rial fibrillation (AF) is an extremely common tachyarrhythmia in humans and terminates either spontaneously or by direct current cardioversion or antiarrhythmic drugs. A long-standing convention holds that under experimental conditions, the termination of $\mathrm{AF}$ is caused by prolongation of the wavelength brought about by prolongation of the atrial effective refractory period and/or depression of atrial conduction! ${ }^{1-3}$ Recently, however, the termination of AF has been attributed to a widening of the excitable gap. In humans, assessment of the atrial intervals on intracardiac electrograms ${ }^{5-9}$ and frequency analysis of surface electrograms ${ }^{10-12}$ and atrial intervals response to drugs, have been performed. Furthermore, the intervals between atrial electrograms during AF have been documented by direct measurement ${ }^{5-7}$ and frequency analysis? ${ }^{7}, 8$

The AF cycle length (AFCL) gradually increases with the administration of class Ia and Ic antiarrhythmic drugs and the atrial electrograms recorded during AF gradually or suddenly become organized prior to the termination of the AF. Recently, some investigators have treated atrial electrograms as random waveforms and analyzed them with the fast Fourier transform (FFT) analysis, 10-12 cross-correlation analysis ${ }^{13}$ or coherence spectrum analysis ${ }^{14}$ The autocorrelation function is a spectrum analysis, known as reversed $\mathrm{FFT}^{15}$ and is a measure of the time-related proper-

(Received May 12, 2003; revised manuscript received July 22, 2003; accepted July 28, 2003)

Division of Cardiovascular Medicine, Yamaguchi University School of Medicine, *Present address: Faculty of Health Sciences, Yamaguchi University School of Medicine, Ube, Japan.

Mailing address: Masunori Matsuzaki, MD, Division of Cardiovascular Medicine, Yamaguchi University School of Medicine, 1-1-1 Minami-kogushi, Ube 755-8505, Japan. E-mail: masunori@po.cc. yamaguchi-u.ac.jp ties of data that are separated by fixed time delays. It can be estimated by delaying the recording relative to itself by some fixed time delay, ${ }^{15} \mathrm{~T}$ and therefore can be used to determine the mean AFCL, as we have previously described 16 There is very little information on the analysis of the atrial electrograms during $\mathrm{AF}$ using the autocorrelation function! 16,17

The AFCL is known to vary temporally as well as spatially. The dispersion expressed as the standard deviation divided by the mean AFCL is one of the methods used to express the variability of the AFCL in time and space $!^{18-23}$ Thus, the aim of the present study was to investigate the termination of $\mathrm{AF}$ by the intravenous administration of cibenzoline using the AFCLs obtained using the autocorrelation function, evaluate the ability of the technique to predict the termination of $\mathrm{AF}$, and ascertain the important factors involved in the termination of AF by cibenzoline infusion.

\section{Methods}

Patients

This protocol was approved by the regional ethics committee and written consent was obtained from all 15 patients enrolled in the study ( 9 males, 6 females, $61 \pm 11$ years old). The clinical characteristics of the subjects are shown in Table 1 . Nine of the 15 patients had a history of paroxysmal AF, which was diagnosed by routine ECG, ambulatory Holter ECG, and bedside monitoring. The patients underwent an electrophysiological study (EPS) as a further investigation of the following conditions: atrioventricular block in 1 patient, syncope in 1, sick sinus syndrome in 2 , ventricular tachycardia in 1 , the treatment of atrial flutter in 7 , atrioventricular nodal reentrant tachycardia in 2 , and 
Table 1 Clinical Characteristics of the Subjects

\begin{tabular}{cccccccc}
\hline $\begin{array}{l}\text { Case } \\
\text { no. }\end{array}$ & $\begin{array}{c}\text { Age } \\
\text { (years) }\end{array}$ & Sex & Diagnosis & $\begin{array}{c}\text { LVEF } \\
(\%)\end{array}$ & $\begin{array}{c}\text { LAD } \\
(\mathrm{mm})\end{array}$ & $\begin{array}{c}\text { Hxof } \\
\text { AF }\end{array}$ & $\begin{array}{c}\text { HXof } \\
\text { AFL }\end{array}$ \\
\hline 1 & 33 & $F$ & AVNRT & 65 & 31 & - & - \\
2 & 57 & $F$ & SSS (III), common AFL & 66 & 26 & + & + \\
3 & 58 & $M$ & WPW syndrome type A & 61 & 17 & - & - \\
4 & 64 & $F$ & SSS (III) & 66 & 51 & + & - \\
5 & 48 & $M$ & PAF, syndrome & 74 & 35 & + & - \\
6 & 56 & $M$ & Idiopathic VT & 68 & 34 & + & - \\
7 & 58 & $M$ & Common AFL & 59 & 41 & - & + \\
8 & 52 & $M$ & AVNRT & 64 & 34 & + & - \\
9 & 77 & $M$ & Common AFL & 77 & 30 & + & + \\
10 & 70 & $F$ & Common AFL & 70 & 40 & + & + \\
11 & 65 & $M$ & AFL & 74 & 35 & + & + \\
12 & 67 & $F$ & Common AFL & 77 & 34 & - & + \\
13 & 65 & $F$ & Common AFL & 69 & 34 & - & + \\
14 & 69 & $M$ & Advanced $A$ block & 69 & 45 & + & + \\
15 & 73 & $M$ & Common AFL & 60 & 45 & - & + \\
\hline
\end{tabular}

$A F$, atrial fibrillation; AFL, atrial flutter, $L V E F$, left ventricular ejection fraction; $L A D$, left atrial dimension; AVNRT, atrioventricular nodal reentrant tachycardia; SSS, sick sinus syndrome; VT, ventricular tachycardia.

Wolff-Parkinson-White syndrome in 1. The EPS was performed while patients were in the fasting state, and all previous antiarrhythmic agents had been discontinued for at least 5 half-lives. Intravenous propofol infusion was used to achieve general anesthesia. AF was induced in each of the 15 patients and then intravenous cibenzoline $(1.4 \mathrm{mg} / \mathrm{kg}$ per $5 \mathrm{~min}$ ) was administered.

\section{Recordings}

A standard $6 \mathrm{~F}$ decapolar catheter with ten 2-mm electrodes with 2-mm interelectrode spacing (Bard Electrophysiolog) was introduced via the right femoral vein and left or right subclavian vein. The catheters were positioned at the high right atrium (HRA), low right atrium (LRA), His bundle and in the coronary sinus (CS). The surface ECG leads were recorded simultaneously with the intracardiac electrograms at a paper speed of $100 \mathrm{~mm} / \mathrm{s}$. Bipolar endocardial electrograms were recorded from each of the 5 closely spaced bipolar pairs of electrodes and filtered through a $30-400 \mathrm{~Hz}$ bandpass filter using a computed electric recorder (EPL00372-001-07, EP Lab, Quinton, Canada).

The atrial electrograms were recorded from the HRA, LRA and CS. For each of the 15 patients in this study, the atrial electrograms during AF were continuously recorded from $30 \mathrm{~s}$ to $60 \mathrm{~s}$ before and during the administration of cibenzoline until either the termination of $\mathrm{AF}$ or $20 \mathrm{~min}$ after the end of the cibenzoline injection if AF had not ceased.

\section{Analysis}

Binary data of the electrograms recorded during AF were retrieved from the hard disk of the EP laboratory system using an optical disk, and then the binary data were transformed using transformation software (BIMUTAS II for Windows, Kissei Comtec, Ltd, Tokyo, Japan) and a Value Star NX personal computer (NEC, Tokyo, Japan) into data suitable for the multi-purpose physio-informatic analysis software (BIMUTAS II for Windows, KISSEI COMTEC). We then selected the transformed data from $30 \mathrm{~s}$ immediately before the intravenous administration of cibenzoline, and immediately before the termination of $\mathrm{AF}$ or the end of the cibenzoline administration if the AF had not ceased within $20 \mathrm{~min}$ after the end of the cibenzoline

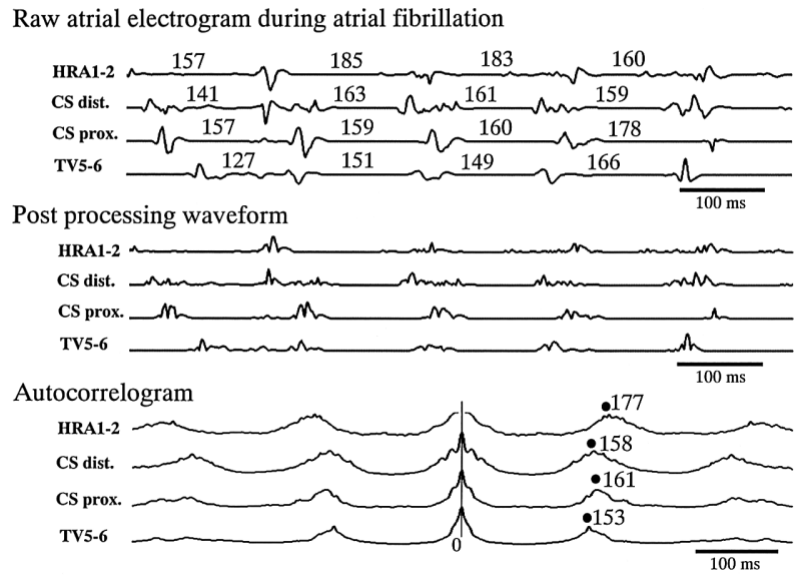

Fig 1. Method of obtaining the peak cycle length of atrial fibrillation (AF). The left side of each panel shows each electrode site. (Upper) A raw atrial electrogram during $\mathrm{AF}$, (Middle) post-processed, rectified electrogram of the same episode in the upper panel, (Lower) autocorrelogram of the same episode in the upper panel. The dot $(\bullet)$ in each autocorrelogram indicates the peak cycle length of AF as shown in the upper panel. The peak cycle length of $\mathrm{AF}$ was retrieved from the first maximum power of the autocorrelofunction on the positive side.

injection.

Autocorrelation analysis for each segment of the recorded electrographic data was performed off-line on a Value Star NX (NEC) using the BIMUTAS II for Windows software package (KISSEI COMTEC) as follows: each set of raw segmental data (upper panel in Fig 1) was subjected to rectification (the post processing waveform in the middle panel in Fig 1) using an installed program in the BIMUTUS II. Each set of rectified data was finally processed to obtain an autocorrelogram (bottom panel in Fig 1) using the BIMUTAS II. The autocorrelation function was defined as:

$$
\operatorname{Rxx}(\tau)=\int_{-\infty}^{\infty} P x x(f) e^{i 2 \pi f \tau} d \tau
$$

$\operatorname{Rxx}(T)$ : autocorrelation function, $\operatorname{Pxx}(f)$ : power spectrum, $\mathbf{T}$ : time displacement, $f$ : cyclical frequency, i: index

The value of the first peak of the coefficient Rxx, on the positive side of the autocorrelogram was taken as the peak $\mathrm{AFCL}^{16}$ and was measured every $5 \mathrm{~s}$ over a $30 \mathrm{~s}$ period 
Table 2 Method of Calculating the Spatial and Temporal Dispersions

\begin{tabular}{|c|c|c|c|c|c|c|}
\hline Time & $0-5 s$ & $5-10 s$ & $10-15 s$ & $15-20 s$ & $20-25 s$ & $25-30 s$ \\
\hline \multicolumn{7}{|l|}{ Site } \\
\hline$H R A$ & Peak AFCL 1 & Peak AFCL 5 & Peak AFCL 9 & Peak AFCL 13 & Peak AFCL 17 & Peak AFCL 21 \\
\hline$C S p$ & Peak AFCL 2 & Peak AFCL 6 & Peak AFCL 10 & Peak AFCL 14 & Peak AFCL 18 & Peak AFCL 22 \\
\hline$C S p$ & Peak AFCL 3 & Peak AFCL 7 & Peak AFCL 11 & Peak AFCL 15 & Peak AFCL 19 & Peak AFCL 23 \\
\hline$L R A$ & Peak AFCL 4 & Peak AFCL 8 & Peak AFCL 12 & Peak AFCL 16 & Peak AFCL 20 & Peak AFCL 24 \\
\hline Average & $A 1$ & $A 2$ & A3 & A4 & A5 & A6 \\
\hline$S D$ & $B 1$ & $B 2$ & B3 & $B 4$ & B5 & B6 \\
\hline Spatial dispersion & $C 1=B 1 \times 100 / A 1$ & $C 2$ & $C 3$ & $C 4$ & $C 5$ & C6 \\
\hline
\end{tabular}

A1-A6 represent the average of the measured AFCLs at each recording site for each $5 \mathrm{~s}$ of data acquisition, and B1-B6 represent their standard deviations, respectively. The spatial dispersion at each timeframe, C1-C6, is defined as (B1-B6) ×100/A1-A6. The mean spatial dispersion is the average of C1 to C6. D1-D4 are the average of each timeframe at each recording site, and E1-E4 represent their standard deviations, respectively. The temporal dispersion at each site, F1-F4, is defined as (D1-D3) ×100/E1-E3. The mean temporal dispersion is the average of F1 to F4.

from the distal pair of electrodes placed in the HRA and LRA, and the distal and proximal pairs of the CS catheter (CSd and CSp, respectively; Fig 1). The average AFCL and standard deviation (SD) were then calculated. Furthermore, the spatial and temporal dispersion were defined as the coefficient of the dispersion $(=\mathrm{SD} \times 100 /$ mean AFCL; Table 2).

The mean AFCL, and the mean spatial and temporal dispersions of the AFCL were compared between the 2 groups of patients formed on the basis of whether or not the AF was terminated by intravenous cibenzoline ( $T$ group: $A F$ was terminated; non-T group: AF was not terminated).

$\mathrm{AF}$ and atrial flutter were defined on the basis of bipolar atrial electrogram recordings using the criteria of Wells et al24,25 AF was defined as a rapid atrial rhythm characterized by variability in the beat to beat cycle length, polarity, configuration and amplitude of the recorded bipolar atrial electrogram 24 Atrial flutter was defined as a regular atrial tachycardia with a stable atrial cycle length $(<20 \mathrm{~ms}$ difference in the cycle lengths of 20-30 consecutive beats) and characteristic sawtooth flutter wave appearance in the inferior ECG leads. 25

All results are mean \pm 1 SD. Paired and unpaired Student's t tests were used for statistical analysis. Statistical significance was established at the $\mathrm{p}<0.05$ level.

\section{Results}

\section{Intravenous Administration of Cibenzoline}

Intravenous cibenzoline terminated $\mathrm{AF}$ in 8 patients (T-group), and did not in 7 patients (non-T group). AF converted to sinus rhythm during the administration of cibenzoline in 3 patients and within 20 min after the end of the cibenzoline administration in 5 patients. AF did not convert to atrial flutter in any patient.

\section{Representative Case}

Fig 2 shows an autocorrelogram from the HRA, CSd and CSp electrodes and the LRA before and immediately before the termination of AF after the cibenzoline administration. The data were taken every $5 \mathrm{~s}$ during the $30 \mathrm{~s}$ of data acquisition before cibenzoline administration and during the $30 \mathrm{~s}$ immediately before the termination of AF. The peak AFCL at each electrode site is marked.

The administration of cibenzoline prolonged the peaked AFCL from $177 \mathrm{~ms}$ to $284 \mathrm{~ms}$ at the HRA, from $158 \mathrm{~ms}$ to $289 \mathrm{~ms}$ at CSd, from $161 \mathrm{~ms}$ to $291 \mathrm{~ms}$ at CSp, and $153 \mathrm{~ms}$ to $293 \mathrm{~ms}$ at the LRA.

Fig 3 showed the time course of the mean AFCL and the mean spatial and temporal dispersions during the administration of cibenzoline in 2 representative cases. The AF shown in the left panel of Fig 3 terminated at $6.5 \mathrm{~s}$ after the administration of cibenzoline. On the other hand, the AF shown in the right panel of Fig 3 did not terminate after cibenzoline, and therefore the data were taken from the 5 min after the end of the administration of cibenzoline.

In the left panel of Fig 3, the mean AFCL (upper panel) and the mean temporal and spatial dispersions are plotted with time. The mean AFCL gradually increased with time, the mean spatial dispersion (solid line) gradually decreased with time, but the change in the mean temporal dispersion (broken line) was unclear.

In the right panel of Fig 3, the mean AFCL gradually increased with time, similar to the case with AF termination shown in the left panel. However, the degree of the increase in the mean AFCL was relatively small, compared with the case with AF termination. The change in the mean spatial and temporal dispersions was unclear.

\section{Mean AFCL, Mean Spatial and Temporal Dispersions}

Intravenous cibenzoline terminated the AF in 8 patients, but not in 7 patients. Cibenzoline significantly increased the AFCL from $182 \pm 15$ to $261 \pm 31 \mathrm{~ms}$ in the T group and from $171 \pm 21$ to $238 \pm 61 \mathrm{~ms}$ in the non-T group (Fig 4). However, the difference from AFCL-before to AFCL-after the administration of cibenzoline between the 2 groups did not reach statistical significance. No significant difference in the AFCL was observed between the T group and non- $T$ group either before or after administration of cibenzoline (left panel in Fig 4).

The mean spatial dispersion significantly $(\mathrm{p}<0.05)$ decreased from $11.1 \pm 9.4$ to $3.2 \pm 1.8$ in the $\mathrm{T}$ group and had a tendency $(\mathrm{p}=0.09)$ to decrease in the non-T group (Fig 4). Note that there was no significant difference in the mean spatial dispersion before cibenzoline, but the mean spatial dispersion after cibenzoline was significantly $(\mathrm{p}<0.05)$ smaller in the $\mathrm{T}$ group than in the non- $\mathrm{T}$ group (middle panel of Fig 4).

The mean temporal dispersion did not significantly decrease with the administration of cibenzoline in either group (right panel of Fig 4). 


\begin{tabular}{ccc}
\hline \hline Average & $S D$ & Temporal dispersion \\
\hline & & \\
$D 1$ & $E 1$ & $F 1=D 1 \times 100 / E 1$ \\
$D 2$ & $E 2$ & $F 2$ \\
$D 3$ & $E 3$ & $F 3$ \\
$D 4$ & $E 4$ & $F 4$ \\
Mean AFCL & Mean temporal SD & Mean Temporal \\
& & dispersion \\
Mean spatial SD & & \\
Mean spatial & & \\
dispersion & & \\
\hline
\end{tabular}

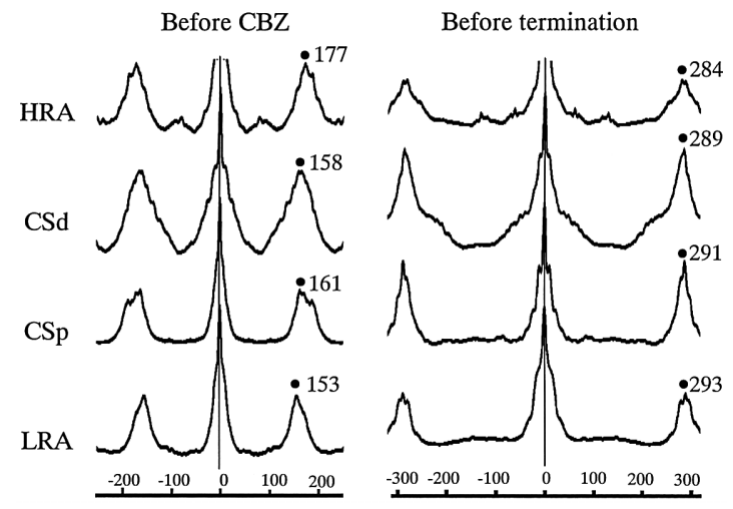

Fig 2. Autocorrelogram in the controls and immediately before the termination of atrial fibrillation (AF). Autocorrelogram before the administration of cibenzoline (Left) and just before the termination of the atrial fibrillation by the administration of cibenzoline (Right). The peak cycle length was prolonged by the administration of the drug.

\section{Discussion}

Recent experimental studies have demonstrated that cardioversion by class I and III drugs cannot be attributed to prolongation of the wavelength, although theoretically the prolongation of the wavelength can certainly lead to termination of $\mathrm{AF}^{26}$ Those studies were based on theoretically accurate measurement of conduction velocity and refractory period during AF using high density mapping,,4 but similar analysis of the termination of human $\mathrm{AF}$ is difficult. Thus, in humans the termination of AF has been observed using either surface electrograms ${ }^{10,11}$ or the measurement of the AA interval during AF ${ }^{-9}$

Bollmann et al performed a frequency analysis of human AF using surface electrograms and the response to ibutilide; they reported that this measurement correlated with the clinical pattern of the arrhythmia and predicted the response to the administration of ibutilide ${ }^{10}$ Slocum et al quantified the frequency content of the fibrillatory baseline as a means to automatically identify $\mathrm{AF}^{26} \mathrm{Holm}$ et al used their data to compare the spatial and temporal dispersions during $\mathrm{AF}^{12}$ To our knowledge, our study is the first to explore the relationship between the peak frequency obtained from the autocorrelation function, and the spatial and temporal dispersions and their responses to the antiarrhythmic drug, cibenzoline.
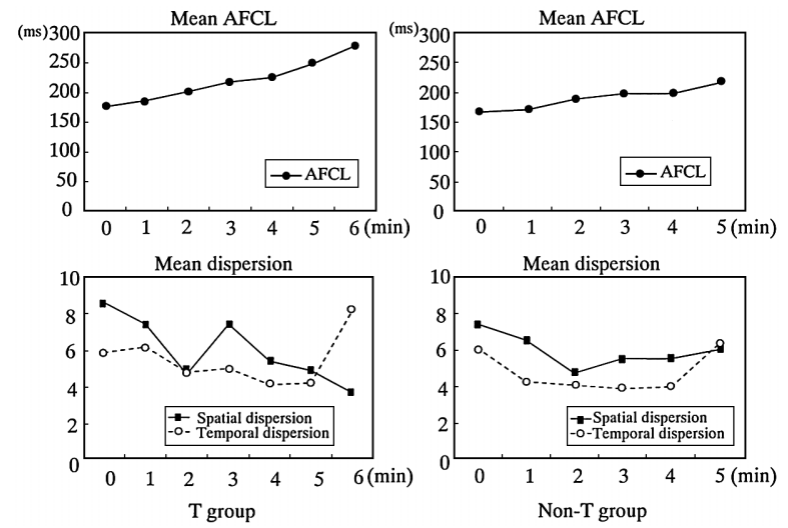

Fig 3. Time course of the mean AFCL, mean spatial dispersion and temporal dispersion of the representative cases.
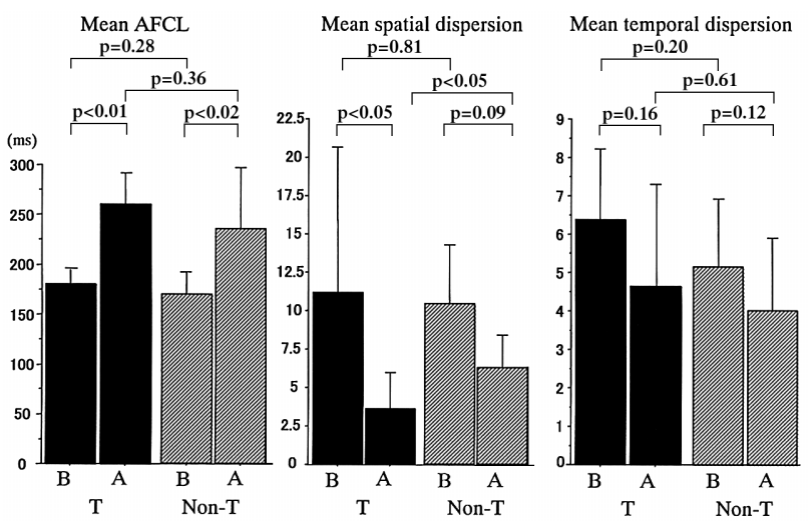

Fig 4. Mean AFCL, and mean spatial and temporal dispersions before and after the administration of intravenous cibenzoline in both the $\mathrm{T}$ group (T) and non-T group (non-T). B, before cibenzoline; A, after cibenzoline; AFCL, atrial fibrillation cycle length.

\section{Measurement of the Peak AFCL and AF Intervals}

Previously, the AF intervals were manually measured to quantitatively estimate the changes ${ }^{27}$ and consequently was a very labor intensive technique. Recently, spectral analysis of the AFCL has been established and estimation of the changes has been attempted using FFT transformation, 8 ,10-12 cross-correlation, ${ }^{13}$ coherence spectrum ${ }^{14}$ and autocorrelofunction analyses ${ }^{16,17}$ Fujiki et al used FFT to investigate the spectral characteristics of human $\mathrm{AF}$ waves with respect to the duration of the $\mathrm{AF}$ and the effect of class I antiarrhythmic drugs (cibenzoline or procainamide); however, they studied the atrial electrogram only from the right atrial free wall and did not estimate the temporal or spatial dispersion?

In the present study, using the computer analysis software, BIMUTUS II, the AFCL was measured with the autocorrelation function and the characteristics of the change in the AFCL were estimated quantitatively when the $\mathrm{AF}$ terminated or did not terminate after administration of an antiarrhythmic drug. One of the advantages of our method is that the peak AFCL pictured by intervals (ms) is more understandable than the cycle length $(\mathrm{Hz})$ pictured by FFT analysis, because we can presume a range of the AFCL. In our method, all the data from the electrograms stored on the hard disk of the EP-LAB during the electrophysiological study were available for analysis. Electro- 
grams sometimes include ventricular electrograms, as can happen when recording close to the AV groove. Because the ventricular cycle length during $\mathrm{AF}$ is much longer (ranging from approximately 600 to $1,000 \mathrm{~ms}$ ) than the atrial cycle length during AF (ranging from approximately 100 to $250 \mathrm{~ms}$ ), this method ignores the ventricular electrograms superimposed on the atrial electrograms during the measurement of the peak AFCL! 16

\section{Mean AFCL, Mean Spatial Dispersion and Mean Temporal \\ Dispersion Before Cibenzoline Administration}

The mechanism of the pharmacological cardioversion of AF is unclear. Fibrillation intervals are known to gradually increase at the termination of AF by antiarrhythmic drugs. The peak AFCL of the local atrium may reflect the local refractoriness, and thus the spatial and temporal dispersions of the AFCL may reflect those of the local refractoriness. In the present study, the mean AFCL before the intravenous administration of cibenzoline was longer in the $T$ group than in the non-T group, but the difference did not reach statistical significance $(\mathrm{p}=0.28)$. In addition, no significant difference in the spatial and temporal dispersions before cibenzoline was observed between the 2 groups. These findings indicate that the mean AFCL, and the spatial and temporal dispersions are not predictive parameters of whether or not the induced AF will be terminated by intravenous cibenzoline.

Some investigators have reported that the mean AFCL can be used to predict whether the AF will be terminated by antiarrhythmic drugs 8,10 However, the period of AF before the drug administration in Fujiki et al's study ${ }^{8}$ was longer than in ours. In the present study, only episodes of induced AF were tested. Thus, the mean AFCL in patients without termination was longer in our study, which may explain why our study did not show a difference in the mean AFCL before cibenzoline between the termination group and nontermination group.

\section{Mean AFCL, Mean Spatial Dispersion and Mean Temporal Dispersion Before Termination by Intravenous Cibenzoline}

In the present study the termination of AF by cibenzoline was marked by an increase in the mean AF cycle length, and a decrease in the spatial and/or temporal dispersion of the AFCL. However, the nature of the most important factor in the termination of human AF by drugs remains unclear.

Boahene et al investigated patients with acute AF and WPW syndrome, and found that the termination of AF was heralded by a $68 \%$ increase in the mean interval of the atrial electrogram after procainamide administration compared with a $30 \%$ increase when the arrhythmia persisted. Thus, they indicated the importance of the AFCL in the termination of AF. In our study, the mean AFCLs in the Tand non- $T$ groups after the intravenous administration of cibenzoline were significantly longer than those before the drug; however, no significant difference in the percentage of prolongation of the mean AFCL was observed between the 2 groups.

In our study, the mean spatial dispersion markedly decreased with time in the T group, whereas it did not significantly change in the non-T group. Thus, the mean spatial dispersion after the administration of cibenzoline was significantly lower in the $\mathrm{T}$ group than in the non- $\mathrm{T}$ group (Fig 4). We emphasize that there were no episodes showing a conversion of AF to atrial flutter in the termination of AF.
Thus, AF unexpectedly terminated to sinus rhythm. Furthermore, cibenzoline did not significantly change the mean temporal dispersion in either the $\mathrm{T}$ group or non- $\mathrm{T}$ group. These findings suggest that the prolongation of the mean AFCL and the decrease in the spatial dispersion are more important factors than the decrease in the temporal dispersion in the termination of AF by cibenzoline.

The average frequency during AF correlates with the wavelength and size of the reentrant pathway ${ }^{28}$ The average size of the reentrant pathway during AF is dependent on the atrial wavelength, defined as the product of the conduction velocity and refractory period 29 Long wavelengths are associated with larger and fewer wavefronts, whereas short wavelengths result in a greater number of small circuits 1,29 The reduction in the average number of fibrillation waves increases the statistical chance of termination of $\mathrm{AF}^{4}$ We speculate that the prolongation of the mean AFCL and the decrease in the spatial dispersion prior to the termination of the AF may reflect larger and fewer wavefronts.

\section{Effects of Propofol on the Electrophysiological Properties in Human Atrial Muscle}

Intravenous propofol infusion was used to achieve general anesthesia during the EPS in our study, because it has been reported that propofol does not have a clinically significant effect on the electrophysiologic expression of the accessory pathway, intraatrial conduction or the refractoriness of the normal $\mathrm{AV}$ conduction system $3^{30}$

\section{Conclusion}

The mean AFCL, and the spatial and temporal dispersions before cibenzoline did not predict the termination of AF. The prolongation of the mean AFCL and the decrease in the spatial dispersion were more important factors than the decrease in the temporal dispersion in the termination of AF by cibenzoline. The decrease in the spatial dispersion may be the important mechanism in the termination of AF by intravenous cibenzoline.

\section{References}

1. Renma PL, Allessie MA, Lammers WJEP, Bonke FI, Schalij MJ. Length of excitation wave and susceptibility to reentrant atrial arrhythmias in normal conscious dugs. Circ Res 1988; 62: 395-410.

2. Wang Z, Page PL, Nattel S. Mechanism of flecanide's antiarrhythmic action in experimental atrial fibrillation. Circ Res 1992; 71: $271-$ 287.

3. Wang J, Bourne GW, Wang Z, Villemaire C, Talajic M, Nattel S. Comparative mechanisms of antiarrhythmic drugs action in experimental atrial fibrillation. Circulation 1993; 88: 1030-1044.

4. Wijffels MCEF, Dorland R, Mast F, Allessie MA. Widening of the excitable gap during pharmacological cardioversion of atrial fibrillation in the goat: The effects of cibenzoline, hydroquinidine, flecainide, and d- sotalol. Circulation 2000; 102: 260-267.

5. Capucci A, Biffi M, Boriani G, Ravelli F, Nollo G, Sabbatani P, et al. Dynamic electrophysiological behavior of human atria during paroxysmal atrial fibrillation. Circulation 1995; 92: 1193-1202.

6. Boahene KA, Klein GJ, Yee R, Sharma AD, Fujimura O. Termination of acute atrial fibrillation in the Wolff-Parkinson-White syndrome by procaninamide and propafenone: Importance of atrial fibrillatory cycle length. J Am Coll Cardiol 1990; 16: 1408-1414.

7. Biffi M, Boriani G, Bronzetti G, Capucci A, Branzi A, Magnani B. Electrophysiological effects of flecainide and propafenone on atrial fibrillation cycle and relation with arrhythmia termination. Heart 1999; 82: 176-182.

8. Fujiki A, Nagasawa H, Sakabe M, Sakurai K, Nishida K, Mizumaki $\mathrm{K}$, et al. Spectral characteristics of human atrial fibrillation waves of the right atrial free wall with respect to the duration of atrial fibrillation and effect of class I antiarrhythmic drugs. Jpn Circ J 2001; 65: $1047-1051$ 
9. Sih HJ, Ropella KM, Swiryn S, Gerstenfeld EP, Sahakian AV, Gerstenfeld EP, et al. Observation from intraatrial recording on the termination of electrically induced atrial fibrillation in humans. Pacing Clin Electrophysiol 1994; 17: 1231 - 1242.

10. Bollmann A, Kanuru NK, McTeague KK, Walter PF, DeLurgio DB, Langberg JJ. Frequency analysis of human atrial fibrillation using the surface electrocardiogram and its response to ibutilide. Am J Cardiol 1998; 81: 1439-1445.

11. Fujiki A, Sakabe M, Nishida K, Mizumaki K, Inoue H. Role of fibrillation cycle length in spontaneous and drug-induced termination of human atrial fibrillation: Spectral anaysis of fibrillation waves from surface electrogram. Circ J 2003; 67: 391-395.

12. Holm H, Pehrson S, Ingemansson M, Sornmo L, Jahansson R, Sandhall $\mathrm{L}$, et al. Non-invasive assessment of the atrial cycle length during atrial fibrillation in man: Introducing, validating and illustrating a new ECG method. Cardiovasc Res 1998; 38: 69-81.

13. Botteron GW, Smith JM. Quantitative assessment of the spatial organization of atrial fibrillation in the intact human heart. Circulation. 1996; 93: 513-518.

14. Ropella KM, Sahakian AV, Baerman JM, Swiryn S. The coherence spectrum: A quantitative discriminator of fibrillatory and nonfibrillatory cardiac rhythms. Circulation 1989; 80: 112-119.

15. Bendat J, Piersol A, editors. Basic descriptions and properties: Random data: Analysis and measurement procedures, 3rd edn. New York: John Wiley \& Sons; 2000: 1-26.

16. Shimizu A, Yoshiga Y, Yamagata T, Esato M, Doi M, Kakugawa H, et al. New method of determining the atrial fibrillation cycle length during human atrial fibrillation. J Cardiovasc Electrophysiol 2003; 14: (in press).

17. Shimizu A, Yamagata T, Ueyama T, Hayano T, Ohmura M, Tanura $\mathrm{K}$, et al. Analysis of atrial electrograms during atrial fibrillation: Autocorrelofunction and interval of atrial electrograms. Jpn $J$ Electrocardiol 1999; 19: 670-678.

18. Lai LP, Lin JL, Lien WP, Tseng YZ, Huang SK. Intravenous sotalol decreases transthoracic cardioversion energy requirement for chronic atrial fibrillation in humans: Assessment of the electrophysiological effects by biatrial basket electrodes. J Am Coll Cardiol 2000; 35: $1434-1441$.

19. Kim KB, Rodefeld MD, Schuessler RB, Cox JL, Boineau JP. Relationship between local atrial fibrillation interval and refractory period in the isolated canine atrium. Circulation 1996; 94: 2961-2967.

20. Rammanna H, Hauer RN, Wittkampf FH, de Bakker JM, Wever EF, Elvan A, et al. Identification of the substrate of atrial vulnerability in patients with idiopathic atrial fibrillation. Circulation 2000; 101: 995-1001.

21. Gaita F, Calo L, Riccardi R, Garberoglio L, Scaglione M, Licciardello G, et al. Different patterns of atrial activation in idiopathic atrial fibrillation: Simultaneous multisite atrial mapping in patients with paroxysmal and chronic atrial fibrillation. J Am Coll Cardiol 2001; 37: 534-541.

22. Rammanna H, Elvan A, Wittkampf FH, de Bakker JM, Hauer RN, Robles de Medina EO. Increased dispersion and shortened refractoriness caused by verapamil in chronic atrial fibrillation. $J$ Am Coll Cardiol 2001; 37: 1403-1407.

23. Zrenner B, Ndrepepa G, Karch MR, Schneider MA, Schreieck J, Schomig A, et al. Electrophysiologic characteristics of paroxysmal and chronic atrial fibrillation in human right atrium. $J$ Am Coll Cardiol 2001; 38: 1143-1149.

24. Wells JL Jr, Karp RB, Kouchoukos NT, MacLean WAH, James TN, Waldo AL. Characterization of atrial fibrillation in man: Studies following open heart surgery. Pacing Clin Elentrophysiol 1978; 1: $426-438$.

25. Stambler BS, Wood MA, Ellenbogen KA. Antiarrhythmic actions of intravenous ibutilide compare with procainamide during human atrial flutter and fibrillation: Electrophysiological determinants of enhanced conversion efficacy. Circulation 1997; 96: 4298-4306.

26. Slocum J, Sahakian A, Swiym S. Diagnosis of atrial fibrillation from surface electrocardiogram based on computer-detected atrial activity. J Electrocardiol 1992; 25: 1-8.

27. Gaita F, Riccardi R, Calo L, Scaglione M, Garberoglio L, Antolini R, et al. Atrial mapping and radiofrequency catheter ablation in patients with idiopathic atrial fibrillation: Electrophysiological findings and ablation results. Circulation 1998; 97: 2136-2145.

28. Konings KT, Kirchhof CJ, Smeets JR, Wellence HJ, Penn OC, Allessie MA. High-density mapping of electrically induced atrial fibrillation in humans. Circulation 1994; 89: 1665-1680.

29. Allessie MA, Bonke FI, Schopman FJ. Circus movement in rabbit atrial muscle as a mechanism of tachycardia: III: The 'leading circle' concept: A new model of circus movement in cardiac tissue without the involvement of an anatomical obstacle. Circ Res 1977; 41: 9-18.

30. Sharpe MD, Dobkowski WB, Murkin JM, Klein G, Yee R. Propofol has no direct effect on sinoatrial node function or on normal atrioventricular and accessory pathway conduction in Wolff-ParkinsonWhite syndrome during alfentanil/midazolam anesthesia. Anesthesiology 1995; 82: 888-895. 